\title{
Optimum shape design of structural model with imprecise coefficient by parametric geometric programming
}

\author{
Samir Dey ${ }^{\mathrm{a}^{*}}$ and Tapan Kumar Roy
}

${ }^{a}$ Department of Mathematics, Asansol Engineering College, Vivekananda Sarani, Asansol-713305, West Bengal, India

${ }^{b}$ Department of Mathematics, Indian Institute of Engineering Science and Technology, Shibpur, P.O.-Botanic Garden, Howrah-711103, West Bengal, India

\begin{tabular}{l}
\hline C H R O N I C L E \\
\hline Article history: \\
Received December 10, 2014 \\
Received in revised format: \\
March 2, 2015 \\
Accepted March 2, 2015 \\
Available online \\
March 2 2015 \\
\hline Keywords: \\
Nonlinear Programming \\
Geometric Programming \\
Structural Optimization \\
Fuzzy number \\
Interval-valued function
\end{tabular}

\begin{abstract}
A B S T R A C T
The article presents solution procedure of geometric programming to solve the structural model with imprecise coefficients. We have considered a single objective structural optimization model with weight as an objective function. Geometric programming provides a powerful tool for solving a variety of imprecise optimization problems. Here we use nearest interval approximation method to convert a triangular fuzzy number to an interval number. In this paper, we transform this interval number to a parametric interval-valued functional form and then solve the parametric problem by geometric programming technique. The advantage of this technique is that we can find directly optimal solution of the objective function without solving two-level mathematical programs. Numerical example is given to illustrate the model through this approximation method.
\end{abstract}

\section{Introduction}

Structural optimization is a critical activity that has received considerable attention in the last four decades. Usually, structural optimization problems involve searching for the minimum of the structural weight. This minimum weight design is subject to various constraints on performance measures, such as stresses and displacements. Optimum shape design of structures is one of the challenging research areas of the structural optimization field. That is why the application of different optimization technique to structural problems has attracted the interest of many researchers. For example, artificial bee colony algorithm (Sonmez, 2011), particle swarm optimization (Luh et al., 2011), genetic algorithm (Dede et al., 2011), ant colony optimization (Kaveh et al., 2010) etc.

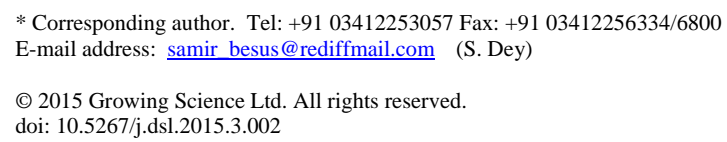


In practice, the problem of structural design may be formed as a typical non-linear programming problem with non-linear objective functions and constraints functions in fuzzy environment. Zadeh (1965) first introduced the concept of fuzzy set theory. Then Zimmermann (1978) applied the fuzzy set theory concept with some suitable membership functions to solve linear programming problem with several objective functions. Some researchers applied the fuzzy set theory to structural model. For example, Wang et al. (1985) first applied $\alpha$-cut method to structural designs where the non-linear problems were solved with various design levels $\alpha$, and then a sequence of solutions were obtained by setting different level-cut value of $\alpha$. Rao (1987) applied the same $\alpha$-cut method to design a four-bar mechanism for function generating problem. Structural optimization with fuzzy parameters was developed by Yeh et al. (1990). Xu (1989) used two-phase method for fuzzy optimization of structures. Shih et al. (2004) used level-cut approach of the first and second kind for structural design optimization problems with fuzzy resources. Shih et al. (2003) developed an alternative $\alpha$-level-cuts methods for optimum structural design with fuzzy resources.

Geometric Programming (GP) method is an effective method used to solve a non-linear programming problem like structural problem. It has certain advantages over the other optimization methods. Here, the advantage is that it is usually much simpler to work with the dual than the primal one. Solving a non-linear programming problem by GP method with degree of difficulty (DD) plays essential role. (It is defined as DD = total number of terms in objective function and constraints - total number of decision variables - 1). Since late 1960's, GP has been known and used in various fields (like OR, Engineering sciences etc.). Duffin et al. (1967) and Zener (1971) discussed the basic theories on GP with engineering application in their books. Another famous book on GP and its application appeared in 1976 (Beightler et al., 1976).The most remarkable property of GP is that a problem with highly nonlinear constraints can be transformed equivalently into a problem with only linear constraints. In real life, there are many diverse situations due to uncertainty in judgments, lack of evidence etc. Sometimes it is not possible to get relevant precise data for the cost parameter. The idea of impreciseness (fuzziness) in GP i.e. fuzzy geometric programming was proposed by Cao (1987). Yang et al. (2010) discussed about the basic and its applications of fuzzy geometric programming. Ojha et al. (2010) used binary number for splitting the cost coefficients, constraints coefficient and exponents and then solved it by GP technique. A solution method of posynomial geometric programming with interval exponents and coefficients was developed by Liu (2008). Nasseri et al. (2014) solved two bar truss nonlinear problem by using geometric programming technique into the form of two-level mathematical programming.

In this paper, we transform interval number to a parametric interval-valued functional form and then structural model becomes parametric structural model, which is solved by geometric programming technique. The proposed procedure is more effective and easy to calculate the different value of the objective function for different value of the parameter. The main benefit of this approximation procedure is that it is not required to create two-level mathematical programming.

\section{Structural Optimization Model}

In sizing optimization problems, the aim is to minimize a single objective function, usually the weight of the structure, under certain behavioral constraints on stress and displacements. The design variables are most frequently chosen to be dimensions of the cross-sectional areas of the members of the structure. Due to fabrication limitations the design variables are not continuous but discrete since crosssections belong to a certain set. A discrete structural optimization problem can be formulated in the following form

$$
\min f(A)
$$

subject to $g_{i}(A) \leq 0, \quad i=1,2, \ldots, m$.

$$
A_{j} \in R^{d}, j=1,2, \ldots ., n \text {. }
$$


where $f(A)$ represents objective function, $g(A)$ is the behavioral constraint, $m$ and $n$ are the number of constraints and design variables, respectively. A given set of discrete values is expressed by $R^{d}$ and design variables $A_{j}$ can take values only from this set.

In this paper, objective function is taken as

$$
f(A)=\sum_{i=1}^{m} \rho_{i} A_{i} l_{i}
$$

and constraints are chosen to be stress of structures as follows,

$$
g_{i}(A)=\frac{\sigma_{i}}{\sigma_{i}^{0}}-1 \leq 0, \quad i=1,2, \ldots, m
$$

where $\rho_{i}$ and $l_{i}$ are weight of unit volume and length of $i^{\text {th }}$ element, respectively, $m$ is the number of the structural elements, $\sigma_{i}$ and $\sigma_{i}^{0}$ are the $i^{\text {th }}$ stress and allowable stress, respectively.

\section{Mathematical Analysis}

\subsection{Geometric Programming}

Geometric program (GP) can be considered as an innovative modus operandi to solve a nonlinear problem in comparison with other nonlinear technique. It was originally developed to design engineering problems. It has become a very popular technique since its inception in solving nonlinear problems. The advantages of this method is that ,this technique provides us with a systematic approach for solving a class of nonlinear optimization problems by finding the optimal value of the objective function and then the optimal values of the design variables are derived. Also this method often reduces a complex nonlinear optimization problem to a set of simultaneous equations and this approach is more amenable to the digital computers. GP is an optimization problem of the form:

$$
\begin{aligned}
& \min g_{0}(x) \\
& \text { subject to } \\
& g_{j}(x) \leq 1, \quad j=1,2, \ldots, m \\
& x_{i}>0 \quad i=1,2, \ldots . ., n
\end{aligned}
$$

where $g_{j}(x)(j=0,1,2, \ldots, m)$ are posynomial or signomial functions , $x$ is decision variable vector of $n$ components $x_{i}(i=1,2, \ldots, n)$.

\subsection{Geometric Programming Problem}

$$
\begin{aligned}
& \min g_{0}(x) \\
& \text { subject to } \\
& g_{j}(x) \leq \delta_{j} b_{j}, \quad(j=1,2, \ldots ., m) \\
& x_{i}>0, \quad(i=1,2, \ldots, n) \\
& \text { where } g_{j}(x)=\sum_{k=1}^{N_{j}} \delta_{j k} c_{j k} \prod_{i=1}^{n} x_{i}^{\alpha_{j k i}} \quad(j=0,1,2, \ldots, m) \\
& \delta_{j}= \pm 1 \quad(j=1,2, \ldots, m), \delta_{j k}= \pm 1\left(j=0,1,2, \ldots, m ; k=1,2, \ldots, N_{j}\right), x \equiv\left(x_{1}, x_{2}, \ldots . ., x_{n}\right)^{T}
\end{aligned}
$$




\subsection{Dual Problem}

The dual problem of the primal problem (5) is as follows, $\max d(w ; \lambda)=\delta_{0}\left(\prod_{j=0}^{m}\left(\frac{c_{j k} w_{j 0}}{w_{j k}}\right)^{\alpha_{j k} w_{j k}}\right)^{\delta_{0}}$ subject to $\sum_{k=1}^{N_{0}} \sigma_{0 k} w_{0 k}=\delta_{0}$ (normal condition)

$$
\sum_{j=0}^{m} \sum_{k=1}^{N_{j}} \delta_{j k} \alpha_{j k i} w_{j k}=0 \quad(i=1,2, \ldots, n) \text { (Orthogonality condition). }
$$

where $\quad \delta_{j}= \pm 1,(j=1,2, \ldots, m), \delta_{j k}= \pm 1\left(j=1,2, \ldots, m ; k=1,2, \ldots, N_{j}\right)$ and $\quad \delta_{0}=+1,-1 \quad$ and $\quad$ nonnegativity conditions, $w_{j 0}=\delta_{j} \sum_{k=1}^{N_{j}} \delta_{j k} w_{j k} \geq 0, w_{j k} \geq 0,\left(j=1,2, \ldots, m ; k=1,2, \ldots, N_{j}\right)$ and $w_{00}=1$.

Case I: For $N \geq n+1$, the dual program presents a system of linear equations for the dual variables where the number of linear equations is either less than or equal to the number of dual variables. A solution vector exists for the dual variable (Beightler et al., 1976).

Case II: For $N<n+1$, the dual program presents a system of linear equations for the dual variables where the number of linear equation is greater than the number of dual variables. In this case, generally, no solution vector exists for the dual variables. However, one can get an approximate solution vector for this system using either the least squares or the linear programming method.

\subsection{Fuzzy number and its nearest interval approximation}

\subsubsection{Fuzzy number}

A real number $\widetilde{A}$ described as fuzzy subset on the real line $\mathfrak{R}$ whose membership function $\mu_{\tilde{A}}(x)$ has the following characteristics with $-\infty<a_{1} \leq a_{2} \leq a_{3}<\infty$

$$
\mu_{\tilde{A}}(x)= \begin{cases}\mu_{\tilde{A}}^{L}(x) & \text { if } a_{1} \leq x \leq a_{2} \\ \mu_{\tilde{A}}^{R}(x) & \text { if } a_{2} \leq x \leq a_{3} \\ 0 & \text { otherwise }\end{cases}
$$

where $\mu_{\tilde{A}}^{L}(x):\left[a_{1}, a_{2}\right] \rightarrow[0,1]$ is continuous and strictly increasing; $\mu_{\tilde{A}}^{R}(x):\left[a_{2}, a_{3}\right] \rightarrow[0,1]$ is continuous and strictly decreasing.

$\alpha$-level set: The $\alpha$-level of a fuzzy number $\widetilde{A}$ is defined as a crisp set $A(\alpha)=\left[x: \mu_{\tilde{A}}(x) \geq \alpha, x \in X\right]$ where $\alpha \in[0,1] . A(\alpha)$ is a non empty bounded closed interval contained in $X$ and it can be denoted by $A_{\alpha}=\left[A_{L}(\alpha), A_{R}(\alpha)\right] . A_{L}(\alpha)$ and $A_{R}(\alpha)$ are the lower and upper bounds of the closed interval ,respectively.

\subsubsection{Interval number}

An interval number $A$ is defined by an ordered pair of real numbers as follows $A=\left[a_{L}, a_{R}\right]=\left\{x: a_{L} \leq x \leq a_{R}, x \in \mathfrak{R}\right\}$ where $a_{L}$ and $a_{R}$ are the left and right bounds of interval $A$, respectively. The interval $A$, is also defined by center $\left(a_{C}\right)$ and half-width $\left(a_{W}\right)$ as follows 
$A=\left\langle a_{C}, a_{W}\right\rangle=\left\{x: a_{C}-a_{W} \leq x \leq a_{C}+a_{W}, x \in \mathfrak{R}\right\}$ where $a_{C}=\frac{a_{R}+a_{L}}{2}$ is the center and $a_{W}=\frac{a_{R}-a_{L}}{2}$ is the half-width of $A$.

\subsubsection{Nearest interval approximation}

Here we want to approximate a fuzzy number by a crisp model. Suppose $\widetilde{A}$ and $\widetilde{B}$ are two fuzzy numbers with $\alpha$-cuts are $\left[A_{L}(\alpha), A_{R}(\alpha)\right]$ and $\left[B_{L}(\alpha), B_{R}(\alpha)\right]$ respectively. Then the distance between $\widetilde{A}$ and $\widetilde{B}$ is

$$
d(\widetilde{A}, \widetilde{B})=\sqrt{\int_{0}^{1}\left(A_{L}(\alpha)-B_{L}(\alpha)\right)^{2} d \alpha+\int_{0}^{1}\left(A_{R}(\alpha)-B_{R}(\alpha)\right)^{2} d \alpha} .
$$

Given $\widetilde{A}$ is a fuzzy number. We have to find a closed interval $C_{d}(\widetilde{A})$, which is the nearest to $\widetilde{A}$ with respect to metric $d$. We can do it since each interval is also a fuzzy number with constant $\alpha$-cut for all $\alpha \in[0,1]$. Hence $\left(C_{d}(\widetilde{A})\right) \alpha=\left[C_{L}, C_{R}\right]$. Now we have to minimize

$$
d\left(\widetilde{A}, C_{d}(\widetilde{A})\right)=\sqrt{\int_{0}^{1}\left(A_{L}(\alpha)-C_{L}\right)^{2} d \alpha+\int_{0}^{1}\left(A_{R}(\alpha)-C_{R}\right)^{2} d \alpha} .
$$

with respect to $C_{L}$ and $C_{R}$.

In order to minimize $d\left(\widetilde{A}, C_{d}(\widetilde{A})\right)$, it is sufficient to minimize the function

$$
D\left(C_{L}, C_{R}\right)\left(=d^{2}\left(\widetilde{A}, C_{d}(\widetilde{A})\right)\right) \text {. The first partial derivatives are }
$$

$$
\frac{\partial}{\partial C_{L}}\left(D\left(C_{L}, C_{R}\right)\right)=-2 \int_{0}^{1} A_{L}(\alpha) d \alpha+2 C_{L} .
$$

and $\frac{\partial}{\partial C_{R}}\left(D\left(C_{L}, C_{R}\right)\right)=-2 \int_{0}^{1} A_{R}(\alpha) d \alpha+2 C_{R}$.

Solving $\frac{\partial}{\partial C_{L}}\left(D\left(C_{L}, C_{R}\right)\right)=0$ and $\frac{\partial}{\partial C_{R}}\left(D\left(C_{L}, C_{R}\right)\right)=0$, we get

$$
C_{L}=\int_{0}^{1} A_{L}(\alpha) d \alpha \text { and } C_{R}=\int_{0}^{1} A_{R}(\alpha) d \alpha .
$$

Again since $\frac{\partial^{2}}{\partial C_{L}^{2}}\left(D\left(C_{L}^{*}, C_{R}^{*}\right)\right)=2>0, \frac{\partial^{2}}{\partial C_{R}^{2}}\left(D\left(C_{L}^{*}, C_{R}^{*}\right)\right)=2>0$ and $H\left(C_{L}^{*}, C_{R}^{*}\right)=\frac{\partial^{2}}{\partial C_{L}^{2}}\left(D\left(C_{L}^{*}, C_{R}^{*}\right)\right) \cdot \frac{\partial^{2}}{\partial C_{R}^{2}}\left(D\left(C_{L}^{*}, C_{R}^{*}\right)\right)-\left(\frac{\partial^{2}}{\partial C_{L} \partial C_{R}}\left(D\left(C_{L}^{*}, C_{R}^{*}\right)\right)\right)^{2}=4>0$.

So $D\left(C_{L}, C_{R}\right)$ i.e. $d\left(\widetilde{A}, C_{d}(\widetilde{A})\right)$ is global minimum. Therefore, the interval

$C_{d}(\widetilde{A})=\left[\int_{0}^{1} A_{L}(\alpha) d \alpha, \int_{0}^{1} A_{R}(\alpha) d \alpha\right]$ is the nearest interval approximation of fuzzy number $\widetilde{A}$ with respect to the metric $d$.

Let $\widetilde{A}=\left(a_{1}, a_{2}, a_{3}\right)$ be a triangular fuzzy number. The $\alpha$-cut interval of $\widetilde{A}$ is defined as $A_{\alpha}=\left[A_{L}(\alpha), A_{R}(\alpha)\right]$ where $A_{L}(\alpha)=a_{1}+\alpha\left(a_{2}-a_{1}\right)$ and $A_{R}(\alpha)=a_{3}-\alpha\left(a_{3}-a_{2}\right)$. By nearest 
interval approximation method the lower limit of the interval is $C_{L}=\int_{0}^{1} A_{L}(\alpha) d \alpha=\int_{0}^{1}\left[a_{1}+\alpha\left(a_{2}-a_{1}\right)\right] d \alpha=\frac{a_{1}+a_{2}}{2}$ and the upper limit of the interval is $C_{R}=\int_{0}^{1} A_{R}(\alpha) d \alpha=\int_{0}^{1}\left[a_{3}-\alpha\left(a_{3}-a_{2}\right)\right] d \alpha=\frac{a_{3}+a_{2}}{2}$.

Therefore, the interval number corresponding $\widetilde{A}$ is $\left[\frac{a_{1}+a_{2}}{2}, \frac{a_{3}+a_{2}}{2}\right]=[m, n]$. In the centre and half width form the interval number of $\widetilde{A}$ is defined as $\left\langle\frac{1}{4}\left(a_{1}+2 a_{2}+a_{3}\right), \frac{1}{4}\left(a_{3}-a_{1}\right)\right\rangle$.

\section{Parametric Interval-valued function}

Let $[m, n]$ be an interval, where $m>0, n>0$. From analytical geometry point of view, any real number can be represented on a line. Similarly; we can express an interval by a function. The parametric interval-valued function for the interval $[m, n]$ can be taken as $g(s)=m^{1-s} n^{s}$ for $s \in[0,1]$, which is strictly monotone, continuous function and its inverse exits. Let $\psi$ be the inverse of $g(s)$, then $s=\frac{\log \psi-\log m}{\log n-\log m}$.

\section{Geometric Programming with fuzzy coefficient}

When all coefficients of Eq. (6) are triangular fuzzy number, then the geometric programming problem is of the form

$$
\min \widetilde{g}_{0}(x)=\sum_{k=1}^{T_{0}} \delta_{0 k} \tilde{c}_{0 k} \prod_{i=1}^{n} x_{i}^{\alpha_{0 k i}}
$$

subject to

$$
\tilde{g}_{j}(x)=\sum_{k=1}^{N_{j}} \delta_{j k} \tilde{c}_{j k} \prod_{i=1}^{n} x_{i}^{\alpha_{j k i}} \leq \delta_{j} \tilde{b}_{j} \text { for } j=1,2,3, \ldots, m .
$$

$x_{i}>0$ for $i=1,2, . ., n$.

where $\tilde{c}_{0 k}=\left(c_{0 k}^{1}, c_{0 k}^{2}, c_{0 k}^{3}\right), \tilde{c}_{j k}=\left(c_{j k}^{1}, c_{j k}^{2}, c_{j k}^{3}\right)$ and $\tilde{b}_{j}=\left(b_{j}^{1}, b_{j}^{2}, b_{j}^{3}\right)$

Using nearest interval approximation method, we transform all triangular fuzzy number into interval number i.e. $\left[c_{0 k}^{L}, c_{0 k}^{U}\right],\left[c_{j k}^{L}, c_{j k}^{U}\right]$ and $\left[b_{j}^{L}, b_{j}^{U}\right]$. The geometric programming problem with imprecise parameters is of the following form

$$
\min \hat{g}_{0}(x)=\sum_{k=1}^{T_{0}} \delta_{0 k} \hat{c}_{0 k} \prod_{i=1}^{n} x_{i}^{\alpha_{0 k i}}
$$

subject to

$\hat{g}_{j}(x)=\sum_{k=1}^{N_{j}} \delta_{j k} \hat{c}_{j k} \prod_{i=1}^{n} x_{i}^{\alpha_{j k i}} \leq \delta_{j} \hat{b}_{j}$ for $j=1,2,3, \ldots, m$.

$x_{i}>0$ for $i=1,2, . ., n$. 
where $\hat{c}_{0 k}, \hat{c}_{j k}$ and $\hat{b}_{j}$ denote the interval counterparts i.e. $\hat{c}_{0 k} \in\left[c_{0 k}^{L}, c_{0 k}^{U}\right], \hat{c}_{j k} \in\left[c_{j k}^{L}, c_{j k}^{U}\right], \hat{b}_{j} \in\left[b_{j}^{L}, b_{j}^{U}\right]$ $, c_{0 k}^{L}>0, c_{j k}^{L}>0, b_{j}^{L}>0$ for all $j$ and $k$. Using parametric interval-valued functional form, the problem (7) reduces to

$\min g_{0}(x ; s)=\sum_{k=1}^{N_{0}} \delta_{0 k}\left(\left(c_{0 k}^{L}\right)^{1-s}\left(c_{0 k}^{U}\right)^{s}\right) \prod_{i=1}^{n} x_{i}^{\alpha_{0 k i}}$

subject to

$$
\begin{aligned}
& g_{j}(x ; s)=\sum_{k=1}^{N_{j}} \delta_{j k}\left(\left(c_{j k}^{L}\right)^{1-s}\left(c_{j k}^{U}\right)^{s}\right) \prod_{i=1}^{n} x_{i}^{\alpha_{j k i}} \leq \delta_{j}\left(\left(b_{j}^{L}\right)^{1-s}\left(b_{j}^{U}\right)^{s}\right) \\
& x_{i}>0 \text { for } i=1,2, . ., n ., j=1,2,3, \ldots, m .
\end{aligned}
$$

This is a parametric geometric programming problem. We get different solutions of this problem for different value of the parameter $s$.

\section{Two Bar Truss Structural Model}

The symmetric two-bar truss (Nasseri et al., 2014) shown in Fig. 1 is considered here. The objective is to minimize the weight of truss subject to the stress $\sigma$ constraints of each bar. There are two design variables- mean tube diameter (d) and height (h) of the truss.

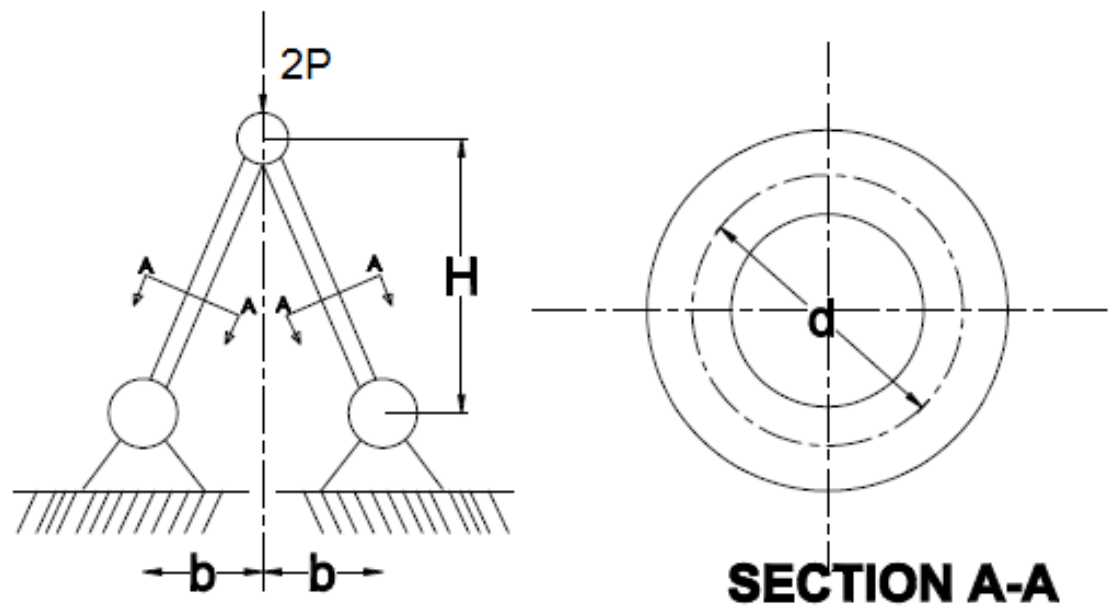

Fig. 1. Two bar truss under load

The weight of the structure is $\rho\left(2 d \pi t \sqrt{b^{2}+h^{2}}\right)$ and stress is $\left(P \sqrt{b^{2}+h^{2}}\right) /(d \pi t h)$. The structural model can be written as

$\min W T(d, h)=\rho\left(2 d \pi t \sqrt{b^{2}+h^{2}}\right)$

subject to

$\sigma(d, h) \equiv \frac{P \sqrt{b^{2}+h^{2}}}{d \pi t h} \leq \sigma_{0}$

$d, h>0$

Let $\sqrt{b^{2}+h^{2}}=y \Rightarrow b^{2}+h^{2}=y^{2}$. Hence the new constraint is $b^{2}+h^{2} \leq y^{2} \Rightarrow b^{2} y^{-2}+h^{2} y^{-2} \leq 1$. Hence the crisp structural model is 
$\min W T(d, h, y)=2 \rho t d \pi y$

subject to

$$
\begin{aligned}
& \sigma(d, h, y) \equiv \frac{P y h^{-1}}{d \pi t} \leq \sigma_{0} \\
& b^{2} y^{-2}+h^{2} y^{-2} \leq 1 \\
& d, h, y>0
\end{aligned}
$$

where $2 \mathrm{P}$ = applied load; $\mathrm{t}=$ thickness of the bar; $\mathrm{d}$ = mean diameter of the bar (decision variable); $2 \mathrm{~b}=$ the distance between two hinge. Model (11) is a standard posynomial geometric programming problem i.e. $\delta_{0}=1, \delta_{01}=1$ and $\delta_{1}=1$. When $\tilde{t}=\left(t_{1}, t_{2}, t_{3}\right), \widetilde{P}=\left(P_{1}, P_{2}, P_{3}\right)$ and $\widetilde{\sigma}_{0}=\left(\sigma_{01}, \sigma_{02}, \sigma_{03}\right)$ are triangular fuzzy number .then the fuzzy structural model is

$\min W T(d, h, y)=2 \tilde{\rho t} d \pi y$

subject to

$$
\begin{aligned}
& \sigma(d, h, y) \equiv \frac{\widetilde{P} y h^{-1}}{d \pi \tilde{t}} \leq \tilde{\sigma}_{0} \\
& b^{2} y^{-2}+h^{2} y^{-2} \leq 1 \\
& d, h, y>0
\end{aligned}
$$

Using nearest interval approximation method, the interval number corresponding triangular number $\tilde{t}=\left(t_{1}, t_{2}, t_{3}\right)$ is $\left[\frac{t_{1}+t_{2}}{2}, \frac{t_{3}+t_{2}}{2}\right]=\left[t_{L}, t_{U}\right]$.Similarly interval number corresponding $\widetilde{P}$ and $\tilde{\sigma}_{0}$ are $\left[\frac{P_{1}+P_{2}}{2}, \frac{P_{3}+P_{2}}{2}\right]=\left[P_{L}, P_{U}\right]$ and $\left[\frac{\sigma_{01}+\sigma_{02}}{2}, \frac{\sigma_{03}+\sigma_{02}}{2}\right]=\left[\sigma_{0 L}, \sigma_{o U}\right]$ respectively. The problem

reduces to

$\min W T(d, h, y)=2 \rho\left[t_{L}, t_{U}\right] d \pi y$

subject to

$$
\begin{aligned}
& \sigma(d, h, y) \equiv \frac{\left[P_{L}, P_{U}\right] y h^{-1}}{d \pi\left[t_{L}, t_{U}\right]} \leq\left[\sigma_{0 L}, \sigma_{0 U}\right] \\
& b^{2} y^{-2}+h^{2} y^{-2} \leq 1 \\
& d, h, y>0
\end{aligned}
$$

which is equivalent to

$\min W T(d, h, y)=2 \hat{\rho t d} \pi y$

subject to

$$
\begin{aligned}
& \sigma(d, h, y) \equiv \frac{\hat{P} y h^{-1}}{d \hat{t}} \leq \hat{\sigma}_{0} \\
& b^{2} y^{-2}+h^{2} y^{-2} \leq 1 \\
& d, h, y>0
\end{aligned}
$$

where $\hat{t} \in\left[t_{L}, t_{U}\right], \hat{P} \in\left[P_{L}, P_{U}\right]$ and $\hat{\sigma}_{0} \in\left[\sigma_{0 L}, \sigma_{o U}\right]$.

\section{Parametric Geometric Programming Technique on Two bar Truss Structural Model}

According to section 4, the fuzzy two bar truss structural model (13) reduces to a parametric programming by replacing $\hat{t}=t_{L}^{1-s} t_{U}^{s}, \hat{P}=P_{L}^{1-s} P_{U}^{s}$ and $\hat{\sigma}_{0}=\sigma_{0 L}^{1-s} \sigma_{0 U}^{s}$ where $s \in[0,1]$ 
The model (14) takes the reduces form as follows

$\min W T(d, h, y)=2 \rho d \pi y\left(t_{L}^{1-s} t_{U}^{s}\right)$

subject to

$$
\begin{aligned}
& \frac{\left(P_{L}^{1-s} P_{U}^{s}\right) y h^{-1}}{d \pi\left(t_{L}^{1-s} t_{U}^{s}\right)\left(\sigma_{0 L}^{1-s} \sigma_{0 U}^{s}\right)} \leq 1 \\
& b^{2} y^{-2}+h^{2} y^{-2} \leq 1 \\
& d, h, y>0
\end{aligned}
$$

Applying Geometric Programming Technique, the dual programming of the problem (14) is

$$
\max g(w)=\left(\frac{2 \pi \rho\left(t_{L}^{1-s} t_{U}^{s}\right)}{w_{01}}\right)^{w_{01}}\left(\frac{\left(P_{L}^{1-s} P_{U}^{s}\right)}{\pi\left(t_{L}^{1-s} t_{U}^{s}\right)\left(\sigma_{0 L}^{1-s} \sigma_{0 U}^{s}\right)}\right)^{w_{11}}\left(\frac{b^{2}}{w_{21}}\right)^{w_{21}}\left(\frac{1}{w_{22}}\right)^{w_{22}}\left(w_{21}+w_{22}\right)^{\left(w_{21}+w_{22}\right)}
$$

subject to

$w_{01}=1$

(Normality condition)

For primal variable $y: 1 \cdot w_{01}+w_{11}+(-2) \cdot w_{21}+(-2) \cdot w_{22}=0 \quad$ (orthogonal condition)

For primal variable $h: 0 \cdot w_{01}+(-1) \cdot w_{11}+0 \cdot w_{21}+2 \cdot w_{22}=0 \quad$ (orthogonal condition)

For primal variable $d: 1 \cdot w_{01}+(-1) \cdot w_{11}+0 \cdot w_{21}+0 \cdot w_{22}=0 \quad$ (orthogonal condition)

$w_{01}, w_{11}, w_{21}, w_{22}>0$

This is a system of four linear equation with four unknowns. Solving we get the optimal values as follows

$w_{01}^{*}=1, w_{11}^{*}=1, w_{21}^{*}=0.5$ and $w_{22}^{*}=0.5$

From primal dual relation we get

$$
\begin{aligned}
& 2 \rho d \pi y\left(t_{L}^{1-s} t_{U}^{s}\right)=w_{01} g^{*}(w) \\
& \frac{\left(P_{L}^{1-s} P_{U}^{s}\right)}{\pi\left(t_{L}^{1-s} t_{U}^{s}\right)\left(\sigma_{0 L}^{1-s} \sigma_{0 U}^{s}\right)} y d^{-1} h^{-1}=\frac{w_{11}}{w_{11}} \\
& b^{2} y^{-2}=\frac{w_{21}}{w_{21}+w_{22}} \\
& h^{2} y^{-2}=\frac{w_{22}}{w_{21}+w_{22}}
\end{aligned}
$$

The optimal solution of the model (14) through parametric approach is given by

$$
\begin{aligned}
& g^{*}(w)=\left(\frac{2 \pi \rho\left(t_{L}^{1-s} t_{U}^{s}\right)}{w_{01}}\right)^{w_{01}}\left(\frac{\left(P_{L}^{1-s} P_{U}^{s}\right)}{\pi\left(t_{L}^{1-s} t_{U}^{s}\right)\left(\sigma_{0 L}^{1-s} \sigma_{0 U}^{s}\right)}\right)^{w_{11}}\left(\frac{b^{2}}{w_{21}}\right)^{w_{21}}\left(\frac{1}{w_{22}}\right)^{w_{22}} \times\left(w_{21}+w_{22}\right)^{\left(w_{21}+w_{22}\right)} \\
& y^{*}=\sqrt{\frac{b^{2}\left(w_{21}+w_{22}\right)}{w_{21}}}, h^{*}=\sqrt{\frac{b^{2} w_{22}}{w_{21}}}, d^{*}=\frac{\left(P_{L}^{1-s} P_{U}^{s}\right)}{\pi\left(t_{L}^{1-s} t_{U}^{s}\right)\left(\sigma_{0 L}^{1-s} \sigma_{0 U}^{s}\right)} \times \sqrt{\frac{b^{2}\left(w_{21}+w_{22}\right)}{w_{21}}} \times \sqrt{\frac{w_{21}}{b^{2} w_{22}}}
\end{aligned}
$$

Note that the optimal solution of GP technique in parametric approach is depends on $s$. 


\section{An illustrative example}

The input data for the structural optimization problem (10) is given as follows:

\begin{tabular}{ccccc}
\hline Load (P) lbs & Thickness (t) in & $\begin{array}{c}\text { Support distance } \\
(2 \mathrm{~b}) \text { in }\end{array}$ & $\begin{array}{c}\text { Material density } \\
(\rho) \mathrm{lbs} / \mathrm{in}^{3}\end{array}$ & $\begin{array}{c}\text { Permissible stress } \\
\left(\sigma_{0}\right) \text { psi }\end{array}$ \\
\hline 33,000 & 0.1 & 60 & 0.3 & 60,000 \\
\hline
\end{tabular}

Formulation of the said model is presented as follows

$\min W T(d, h, y)=0.188 y d$

subject to

$$
\begin{aligned}
& 1.75 y d^{-1} h^{-1} \leq 1 \\
& 900 y^{-2}+h^{2} y^{-2} \leq 1 \\
& d, h, y>0
\end{aligned}
$$

This is a Posynomial Geometric Programming Problem with degree of difficulty $(D D)=4-(3+1)=0$.

Applying Geometric Programming Technique, the dual programming of the problem (16) is

$$
\max g(w)=\left(\frac{0.188}{w_{01}}\right)^{w_{01}}(1.75)^{w_{11}}\left(\frac{900}{w_{21}}\right)^{w_{21}}\left(\frac{1}{w_{22}}\right)^{w_{22}}\left(w_{21}+w_{22}\right)^{\left(w_{21}+w_{22}\right)}
$$

subject to

(Normality condition)

$w_{01}=1$

For primal variable $y: 1 \cdot w_{01}+w_{11}+(-2) \cdot w_{21}+(-2) \cdot w_{22}=0$

(orthogonal condition)

For primal variable $h: 0 \cdot w_{01}+(-1) \cdot w_{11}+0 \cdot w_{21}+2 \cdot w_{22}=0$ (orthogonal condition)

For primal variable $d: 1 \cdot w_{01}+(-1) \cdot w_{11}+0 \cdot w_{21}+0 \cdot w_{22}=0$ (orthogonal condition)

$$
w_{01}, w_{11}, w_{21}, w_{22}>0
$$

This is a system of four linear equation with four unknowns. Solving we get the optimal values as follows

$$
w_{01}^{*}=1, w_{11}^{*}=1, w_{21}^{*}=0.5 \text { and } w_{22}^{*}=0.5
$$

From primal dual relation we get

$$
0.188 y d=w_{01} g^{*}(w), 1.75 y d^{-1} h^{-1}=\frac{w_{11}}{w_{11}}, 900 y^{-2}=\frac{w_{21}}{w_{21}+w_{22}} \text { and } h^{2} y^{-2}=\frac{w_{22}}{w_{21}+w_{22}}
$$

Solving this we get the optimum solution of the problem (16) by Geometric Programming (GP) Technique is presented in Table 1

\section{Table 1}

Optimal solution of Two Bar Truss Structural Model

\begin{tabular}{ccccc}
\hline Method & Weight WT $^{*}(\mathrm{lbs})$ & $\begin{array}{c}\text { Diameter } \\
\mathrm{d}^{*}(\text { in) }\end{array}$ & $\begin{array}{c}\text { Height } \\
\mathrm{h}^{*}(\mathrm{in})\end{array}$ & $\mathrm{y}^{*}$ (in) \\
\hline GP & 19.74 & 2.474874 & 30 & 42.426402 \\
NLP & 19.74 & 2.474874 & 30 & 42.42641 \\
Schmit (1981) & 19.8 & 2.47 & 30 & -------- \\
\hline
\end{tabular}


When the input data of two bar truss structural model (11) is taken as triangular fuzzy number i.e. $\widetilde{P}=(28000,32000,34000), \tilde{t}=(0.06,0.08,0.12) \quad$ and $\quad \tilde{\sigma}_{0}=(55000,59000,61000)$. Using nearest interval approximation method , we get the corresponding interval number and interval-valued function i.e. $\quad \mathrm{P}=[30000,33000], \Rightarrow \quad \hat{\mathrm{P}}=(30000)^{1-\mathrm{s}}(33000)^{\mathrm{s}} \in[30000,33000], \quad \mathrm{t}=[0.07,0.1]$, $\Rightarrow \hat{t}=(0.07)^{1-s}(0.1)^{s} \in[0.07,0.1]$ and $\sigma_{0}=[57000,60000]$ $\Rightarrow \hat{\sigma}_{0}=(57000)^{1-s}(60000)^{s} \in[57000,60000]$ where $s \in[0,1]$

The optimal solution of the fuzzy model by interval-valued parametric geometric programming is presented in Table 2.

Table 2

Optimal solution of Two Bar Truss Structural Model

\begin{tabular}{ccccc}
\hline $\mathrm{s}$ & $\begin{array}{c}\text { Weight } \\
\mathrm{WT}^{*}(\mathrm{lbs})\end{array}$ & $\begin{array}{c}\text { Diameter } \\
\mathrm{d}^{*}(\mathrm{in})\end{array}$ & $\begin{array}{c}\text { Height } \\
\mathrm{h}^{*}(\mathrm{in})\end{array}$ & $\mathrm{y}^{*}(\mathrm{in})$ \\
\hline 0.0 & 18.94736842 & 3.386364548 & 30 & 42.42640687 \\
0.1 & 19.03952650 & 3.282125186 & 30 & 42.42640687 \\
0.2 & 19.11490561 & 3.181094530 & 30 & 42.42640687 \\
0.3 & 19.19922892 & 3.083173808 & 30 & 42.42640687 \\
0.4 & 19.28392421 & 2.988267290 & 30 & 42.42640687 \\
0.5 & 19.36899312 & 2.896282192 & 30 & 42.42640687 \\
0.6 & 19.45443731 & 2.807128588 & 30 & 42.42640687 \\
0.7 & 19.54025843 & 2.720719317 & 30 & 42.42640687 \\
0.8 & 19.62645813 & 2.626969904 & 30 & 42.42640687 \\
0.9 & 19.58172288 & 2.555798472 & 30 & 42.42640687 \\
1 & 19.80000000 & 2.477125667 & 30 & 42.42640687 \\
\hline
\end{tabular}

For $s=0$, the lower bound of the interval value of the parameter is used to find the optimal solution. For $s=1$, the upper bound of interval value of the parameter is used for the optimal solution. These results yield the lower and upper bounds of the optimal solution. The main advantage of the proposed technique is that one can get the intermediate optimal result using proper value of $s$.

\section{Conclusion}

The advantage of this technique is that we can find directly optimal solution of the objective function without solving two-level mathematical programs. This method is simple and takes minimal time. Here decision maker (engineer) may obtain the optimum results as per his/her requirement .The methodology presented in this paper can be applied in other fields of engineering optimization.

\section{Acknowledgements}

The authors would like to thank the anonymous referees for constructive comments on earlier version of this paper. The great support of my family members makes me possible to do it.

\section{Reference}

Wang, G.Y. \& Wang, W.Q. (1985). Fuzzy optimum design of structure. Engineering Optimization, 8, 291-300.

Zadeh, L.A. (1965). Fuzzy set. Information and Control, 8(3), 338-353.

Zimmermann, H.J. (1978).Fuzzy linear programming with several objective function .Fuzzy sets and systems, 1 , 45-55. 
$\mathrm{Xu}, \mathrm{C}$. (1989).Fuzzy optimization of structures by the two-phase method. Computer and Structure, 31(4),575-580.

Yeh, Y.C. \& Hsu, D.S. (1990).Structural optimization with fuzzy parameters. Computer and Structure,37(6), 917-924.

Rao, S.S. (1987). Description and optimum design of fuzzy mathematical systems. Journal of mechanical design,109(1), 126-132.

Shih,C. J. \& Lee, H. W.(2004).Level-cut Approaches of First and Second Kind for Unique Solution Design in Fuzzy Engineering Optimization Problems. Tamkang Journal of Science and Engineering, 7(3),189-198.

Shih,C.J., Chi,C.C. \& Hsiao,J.H.(2003).Alternative $\alpha$-level-cuts methods for optimum structural design with fuzzy resources, Computers and Structures, 81,2579-2587.

Kaveh, A. \& Talatahari, S. (2010).An improved ant colony optimization for the design of planar steel frames. Engineering Structure, 32(3), 864-873.

Dede, T., Bekiroğlu, S. \& Ayvaz, Y. (2011).Weight minimization of trusses with genetic algorithm. Applied Soft Computing ,11(2), 2565-2575.

Luh, G.C. \&Lin, C.Y.(2011). Optimal design of truss-structures using particle swarm optimization. Computers and Structures, 89(2324), 2221-2232.

Sonmez, M. (2011).Discrete optimum design of truss structures using artificial bee colony algorithm. Structural and multidisciplinary optimization, 43(1),85-97.

Yang, H. \& Cao, B. Y.(2010). Fuzzy geometric programming and its application. Fuzzy information and engineering, 2(1), 101-112.

Zener, C. (1971). Engineering design by geometric programming, Wiley, New York.

Beightler,C.S.\& Phillips, D.T. (1976).Applied geometric programming. Wiley, New York .

Duffin,R.J., Peterson,E.L. \& Zener,C.M. (1967).Geometric programming- theory and applications. Wiley, New York.

Schmit, L.A. (1981). Structural synthesis- its genesis and development. AIAA Journal, 119(10), 12491263.

Ojha, A. K. \& Das, A.K. (2010).Geometric programming problem with coefficients and exponents associated with binary numbers. International Journal of Computer Science Issues, 7(1), 49-55.

Liu, S. T. (2008). Posynomial geometric programming with interval exponents and coefficients. European Journal of Operational Research, 186(1), 17-27.

Cao, B.Y. (1987). Solution and theory of question for a kind of fuzzy positive geometric program. Proceeding of the second IFSA Congress, Tokyo, 1, 205-208.

Nasseri, S. H. \& Alizadeh, Z. (2014). Optimized solution of a two-bar truss nonlinear problem using fuzzy geometric programming. Journal of Nonlinear Analysis and Application, 1-9. doi:10.5899/2014/jnaa-00230 